\title{
Massive ocular astrocytoma in a congenitally deformed eye
}

\author{
J. REIMER WOLTER, ${ }^{3}$ ALMON L. SCHUT, ${ }^{2}$ AND KATERINA DOROVINI-ZIS ${ }^{3}$
}

From the Departments of 'Ophthalmology and ${ }^{3}$ Pathology of the University of Michigan Medical Center, and from the ${ }^{2}$ Bronson Methodist Hospital, Kalamazoo, USA

SUMMARY A large retinal astrocytoma filled all of the posterior chamber of the congenitally blind and deformed eye of a 26-year-old male. History, clinical findings, and pathological study with special stains allowed for an unusually complete presentation of this rare intraocular neoplasm.

Astrocytomas of the inner eye are rare neuroectodermal hamartomas. ${ }^{12}$ They may originate in the retina $^{3}$ or in the optic nerve head. ${ }^{4}$ Their advanced stages are usually found in the laboratory in eyes that were removed because of pain and blindness, ${ }^{3}$ but in their early stages they may present to the clinician with all the signs of active intraocular tumour growth ${ }^{\prime}$ and may be clinically indistinguishable from retinoblastoma. ${ }^{2}$ Like other extracerebral gliomas in the head region intraocular astrocytomas typically are benign. ${ }^{5}$ Gliomatous tumours in the eye present in a broad and continuous spectrum of space-taking processes showing excessive reactive gliosis on one end and active neoplastic glial growth on the other. ${ }^{3}$ The reactive tumours are also known as massive gliosis and are relatively common. True intraocular gliomas are exceedingly rare. An attempt to find features that would allow for an exact separation of massive gliosis from the neoplastic types in a larger series has failed. ${ }^{3}$ All these glial tumours occur in patients who show no signs of generalised phakomatoses, of course.

It is the purpose of this paper to present the case of a patient with an astrocytoma that completely filled a congenitally deformed eye and extended into the optic nerve head, and who had a slight congenital defect in the other eye.

\section{Case report}

This 26-year-old white male was first seen in March 1978 with a blind, painful, and unsightly left eye. The family history was negative, but it may be of interest that the patient was the fifth of 5 living siblings and that

Correspondence to J. Reimer Wolter. MD. Department of Ophthalmology. University of Michigan Medical Center. Ann Arbor, Michigan 48109. USA. one sister died at the age of 1 month, while another sister was stillborn. The patient was born of a normal pregnancy, at full term by a normal delivery, and was not in an incubator. He is of good general health and stated that he had never been sick. He has no signs of neurofibromatosis or other generalised disease. The left eye was recognised as poorly developed at birth and it never had any vision. Throughout his childhood it did not bother the patient, but within the last 2 years it became painful and ugly.

Clinical examination revealed a vision of $20 / 20$ and $\mathrm{J} \mathrm{O}$ with a $-4.00+0.50$ axis $180^{\circ}$ in the right eye. This eye had a normal visual field. Fundus examination of the right eye revealed a relatively large temporal conus of the disc, with an anomalous choroidal blood vessel crossing the region of the conus. The left eye was blind. It was irritable and also exhibited band keratopathy as well as dense corneal scarring, which prevented a view into deeper parts of the eye; it appeared to be small. In 1967 the eye had a low intraocular pressure. In 1976 an irregular staphyloma was noticed on the temporal side between 2 and 6 o'clock. At that time, the intraocular pressure was $28.0 \mathrm{mmHg}$ by applanation tonometry. There was distinct rubeosis iridis. After the situation had been discussed with the patient he asked to have the eye removed. This was done on 22 March 1978 under general anaesthesia.

The eye measured $22 \times 19 \times 19 \mathrm{~mm}$. The cornea was white and scar-like. There was an extensive staphyloma, with bluish discolouration of the sclera. Transillumination with the egg candler ${ }^{6}$ showed the eye to be filled with a solid mass. When the eyeball was opened in a horizontal plane, the anterior chamber could be recognised and was partly filled with whitish exudate. The iris was continuous, and its front surface had a normal appearance. A white 
Fig. 1 Gross view of the eye after sectioning. The anterior chamber (A) filled with a yellowish substance. The iris is visible. The inner eye behind the iris is totally filled with a white tumour $(\mathrm{T})$. $(\times 2 \cdot 8)$.

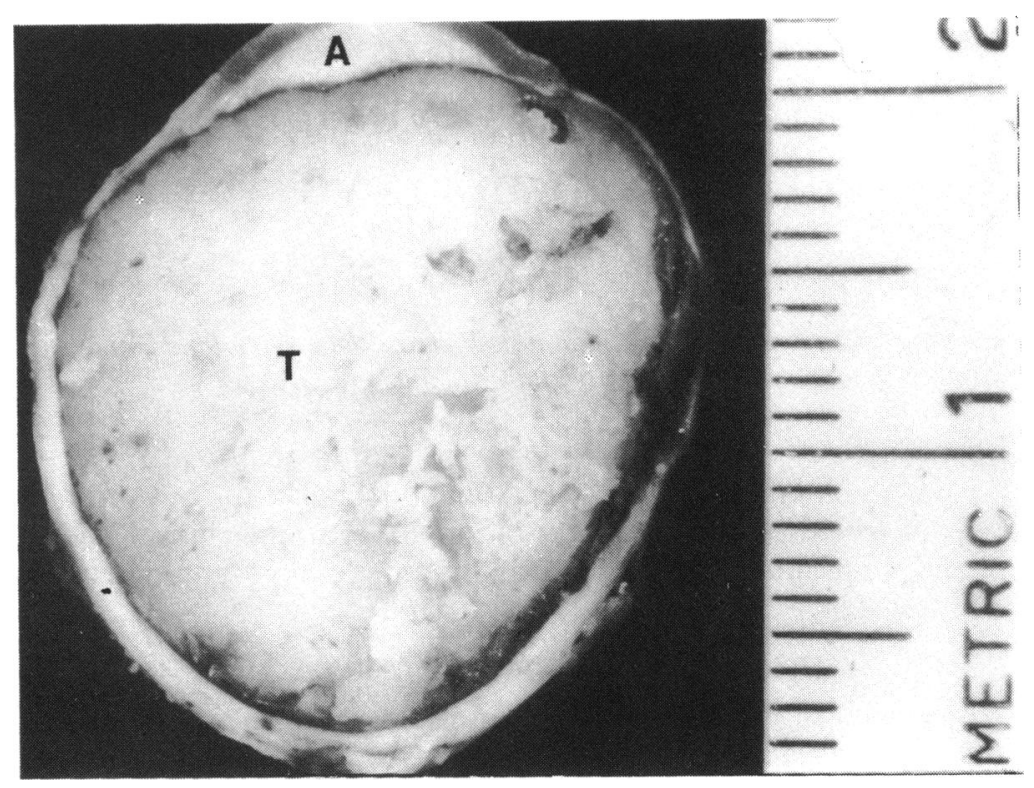

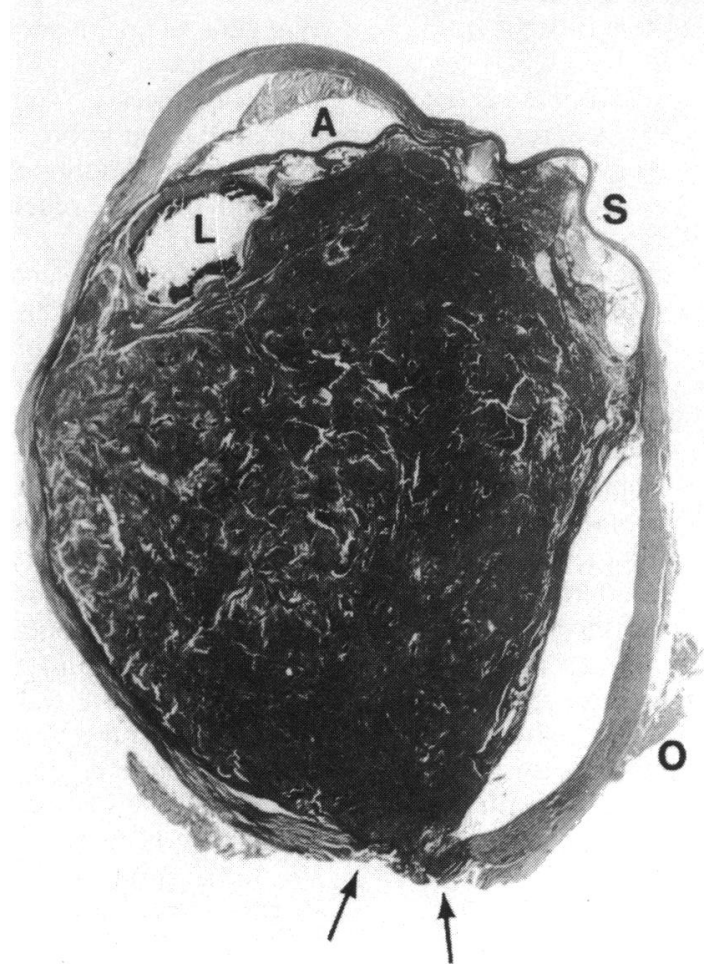

Fig. 2 Cross-section of the eye showing exudate in the anterior chamber (A), the lens (L) with much of the lens substance broken out because of calcification, the staphyloma on the right (S), the tumour filling all of the inner eye behind the iris, and the hole in the sclera posteriorly (between arrows). The inferior oblique insertion is seen on the sclera (O). (Paraffin section, $H$ and $E$ stain, $\times 3 \cdot 4)$. tumour of firm consistency filled the remainder of the eye (Fig. 1). In the place of the optic nerve the posterior sclera had a hole.

Microscopic study of paraffin sections (Fig. 2) revealed a continuous corneal epithelium of somewhat irregular thickness, with a dense fibrous pannus containing only a few patent blood vessels. Bowman's membrane was seen under the pannus in partly calcified fragments. The corneal stroma showed much scarring and some neovascularisation. The anterior chamber was shallow, and it was partly filled with serous exudate containing cholesterol crystal spaces and many histiocytes. Anterior peripheral synechiae closed the angle completely. The iris was atrophic and showed distinct neovascularisation, with relatively large vessels. The pupil was not recognised. A staphyloma was seen in the region of the ciliary body on one side (Fig. 2); the lens was pushed to the opposite side and was calcified. Tumour tissue filled the remainder of the inner eye. Parts of atrophic and distorted retina were seen in their normal place, but most of the retina was absent. The pigment epithelium had survived in some parts, but most of this layer had also degenerated and had formed a thin layer of bone overlying remnants of the choroid. The choroid was not directly involved with the tumour. Mononuclear cell infiltration was recognised, both diffusely and in a focal arrangement in the choroid. The optic nerve head was not seen, but the tumour extended through the hole cut during enucleation in the sclera in the place of the optic nerve (Fig. 2). The sclera was otherwise normal, though it had unusually large, long posterior ciliary nerves.

The tumour filling most of the inner eye had a 


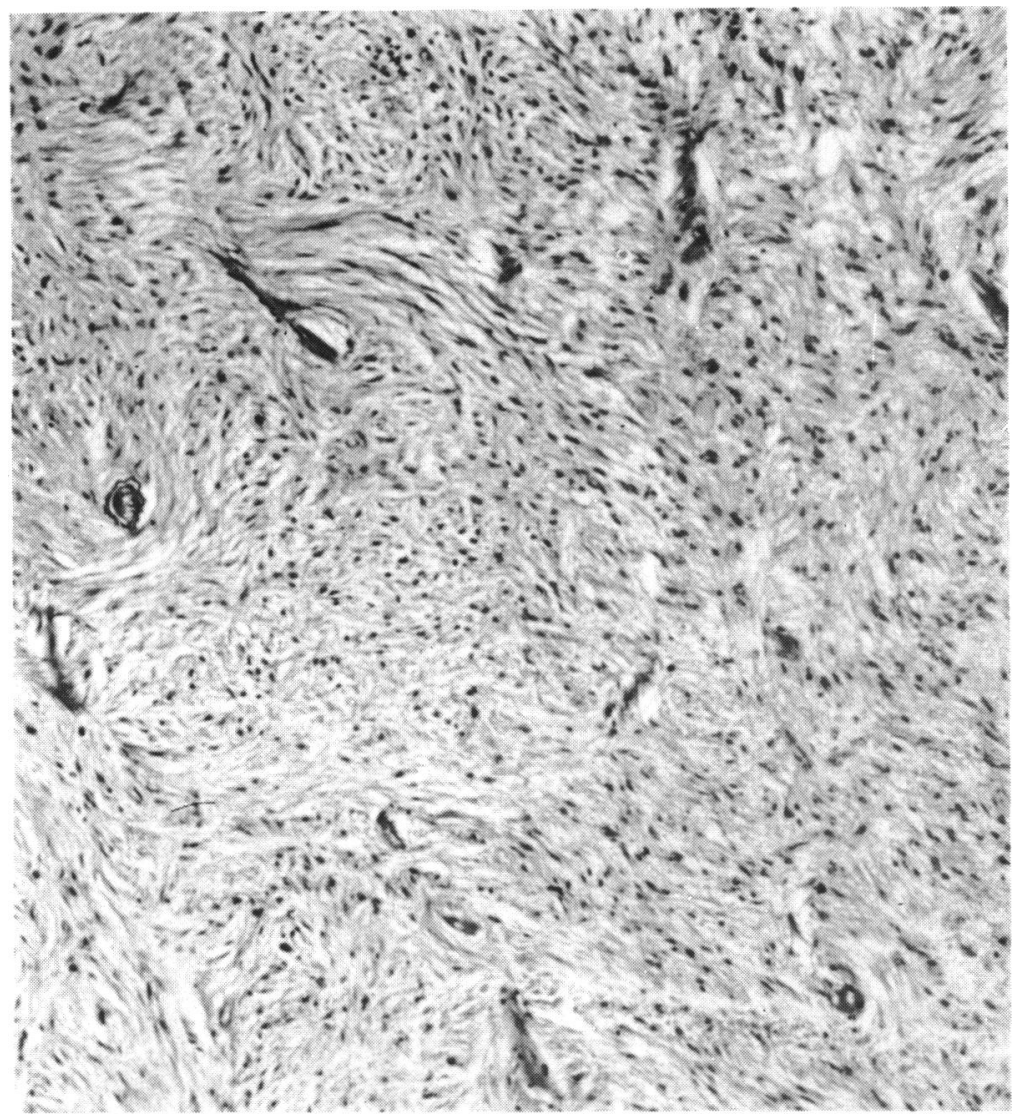

Fig. 3 Tumour cells are arranged in interlacing bundles. They are quite uniform and exhibit ovoid or elongated nuclei. Numerous blood vessels with hyalinised walls are seen. (Thin paraffin section, $H$ and Estain, $\times 300$ ).

slightly irregular appearance due to a poorly developed lobulated growth pattern. This was best visible at lower magnifications (Fig. 2). Higher power showed it to be composed of intertwining bundles of elongated bipolar cells with small and oval nuclei. All the cells appeared rather inactive, and no mitoses were found. Blood vessels were seen all through the tumour in a fairly regular distribution and their walls showed much hyalinisation (Fig. 3). Small areas of necrosis were found, and these showed early stages with necrotic preservation of portions of fibre bundles as well as later stages with hyalinisation and calcification. Phosphotungstic acid haematoxylin (PTAH) stain revealed fine and coarse neuroglial fibres in the tumour cells. Occasionally the cells were arranged radially around a small lumen to form rosettes (Fig. 4). Rosenthal fibres similar to those observed in pilocytic astrocytomas of the central nervous system were seen in places (Fig. 5). Reticulum stain showed only few reticulum fibres, which were associated with the blood vessels. The cytoplasm and the processes of the tumour cells failed to stain red with the Gomori trichrome stain. Electron microscopic examination of the formalin-fixed tissues showed numerous intracytoplasmic filaments. Unfortunately the preservation was not sufficient to allow for further identification of the cells and their filaments. However, the observations with special stains and light microscopy were sufficient to make the diagnosis of a retinal astrocytoma in this case.

Study of the posterior aspect of the enucleated eye with the scleral hole resulted in the impression that the astrocytoma was not completely removed. Thus on 15 May 1978 conjunctiva and Tenon's capsule were opened, the mesh implant was temporarily removed, and the optic nerve head was excised along with a $7 \mathrm{~mm}$ piece of the optic nerve stump. Histological study revealed additional astrocytoma on the lamina cribrosa, but the tumour had not extended into the nerve. The optic nerve was atrophic. The central retinal artery exhibited advanced narrowing due to an onion-layer-like proliferation of the endothelium (Fig. 6). The central retinal vein was large, but its lumen was filled with organised thrombus. Again, the ciliary nerves around the optic nerve were observed to be unusually large. 


\section{Discussion}

Only a few well documented cases of retinal astrocytomas $^{2}$ and one case of astrocytoma of the optic nerve head ${ }^{4}$ have been reported. The special value of the present case is in its history and some objective findings, which indicate its congenital nature. Associated with a slightly anomalous optic nerve head on the other side, the involved eye in the present case comes with a good history of being obviously abnormal and blind since birth. Unusually large ciliary nerves also indicate a primary neuroectodermal anomaly. However, study of the eye with its fully developed cornea, lens, sclera, choroid, portions of retina, and optic nerve indicates that it was relatively well developed before it became blind. The history, clinical observations, and pathological findings fit the picture of an eye that became involved with an intraocular tumour early in life-possibly before birth. This tumour slowly enlarged and filled the eye. It is impossible to say exactly where in the eye it originated, but the nasal displacement of the lens and the temporal staphyloma suggest the retinal periphery on the temporal side of the eye as a likely site of origin. The tumour was noninvasive, and thus it did not break the barriers of iris, Bruch's membrane, and lamina cribrosa. It caused posterior bulging of th lamina cribrosa, however. Its slow growth attracted most of the intraocular blood supply, and it caused atrophy of all other intraocular structures by compression and additional ischaemia. The ischaemia. finally, caused rubeosis, and the resulting secondary glaucoma led to pain and enucleation. The final stage, with rubeosis and secondary glaucoma, is common in cases with slowly growing intraocular tumours, of course.

As in all accepted cases of intraocular astrocytomas the tumour in the present case was not associated with any evidence of neurofibromatosis or another phakomatosis. The distinctly enlarged ciliary nerves.

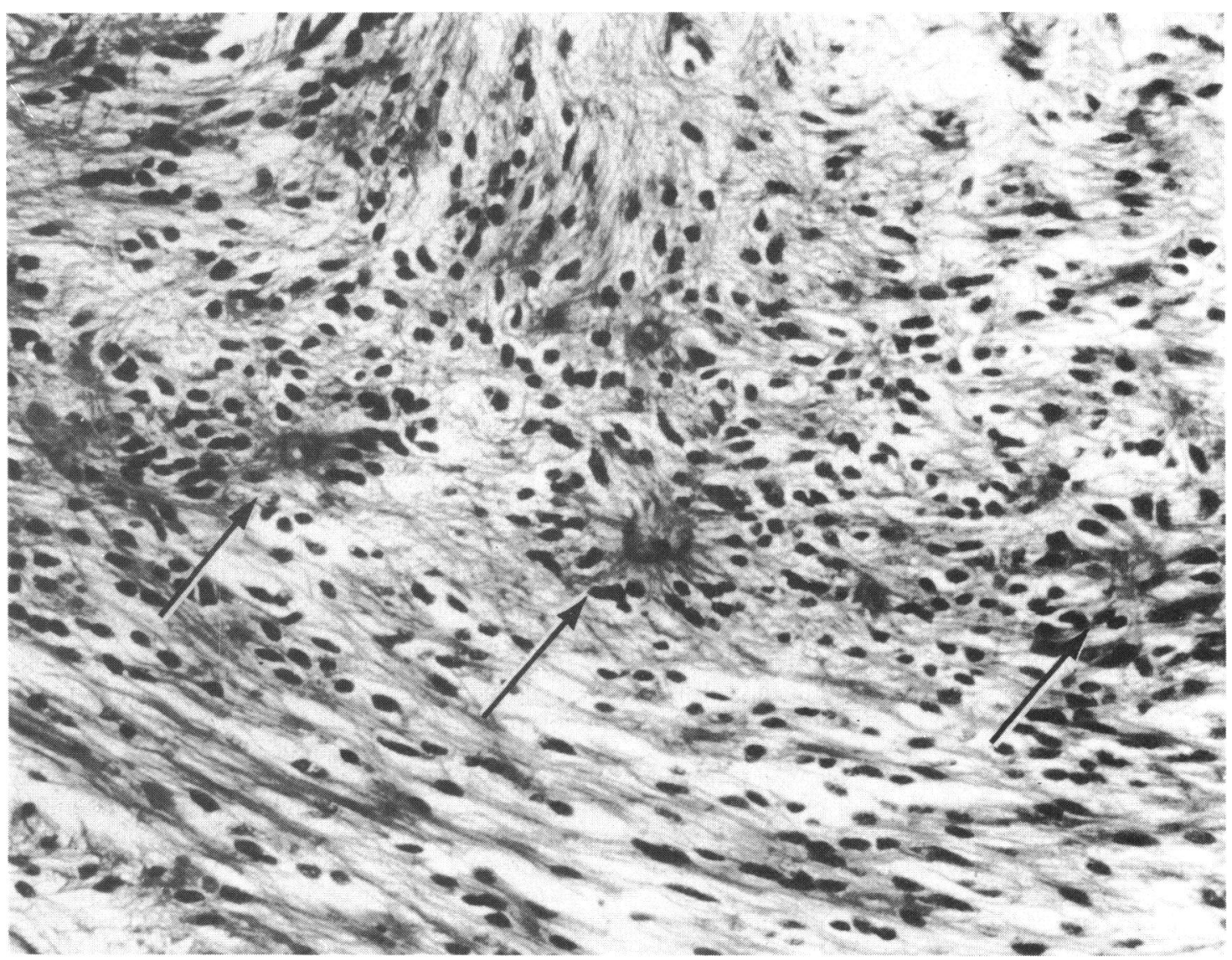

Fig. 4 Occasionally the tumour cells assemble to form rosettes (arrows). Their nuclei are peripherally located and long cytoplasmic processes project to a central lumen. (Paraffin section, $H$ and $E$ stain, $\times 300$ ). 


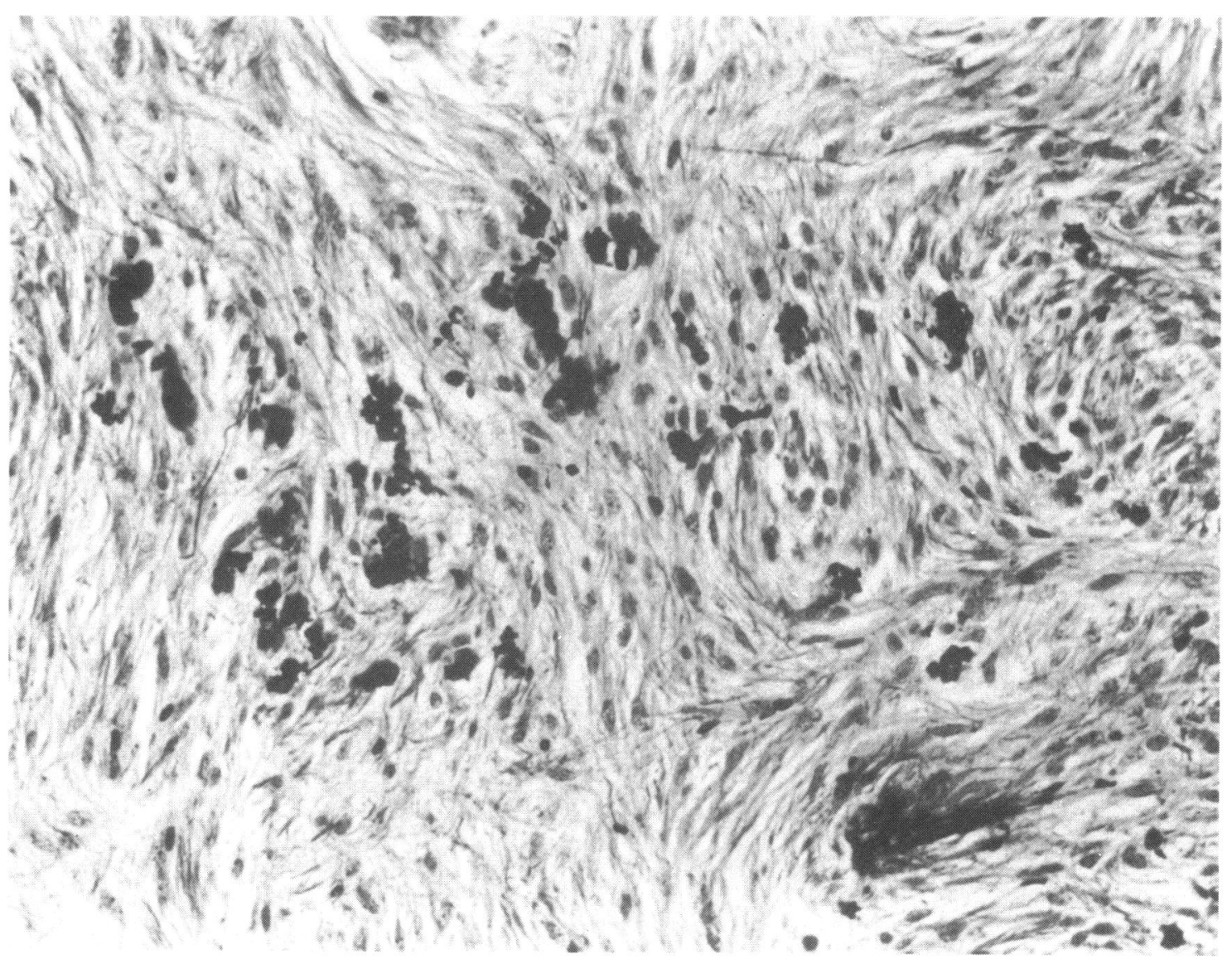

Fig. 5 Darkly-stained Rosenthal fihres in cells of the present tumour. (Paraffin section. PTAH stain. $\times 3(00)$.

however. are evidence of an overabundance of uveal nerves. which has been seen only as a congenital condition ${ }^{7}$ and not as a reaction to chronic eve disease. This is found in some cases of choroidal melanoma-often part of associated overabundance of melanocytes and primitive ganglion cells and definitely related to so-called ocular melanosis. Uveal changes of this kind have been recognised as a congenital predisposition for the development of malignant uveal melanomas. ${ }^{89}$ The significance of the large posterior ciliary nerves in the present case is not clear. but their presence is a sign of a congenital anomaly with excessive tissue of neuroectodermal origin. As in other hamartomas. the development of a retinal astrocytoma would probably originate in primitive glial elements left over from early development rather than from fully differentiated glial cells of the retina. ${ }^{10}$

The present case, thus, not only presents with an unusually advanced stage of an extremely rare and easily demonstrable intraocular tumour, but it also offers some indications that the tumour developed very early in life on the basis of a congenital anomaly. These facts are important additions to our knowledge not only of intraocular gliomas but also of principles concerning the nature and growth of ocular neoplasms in general.

This work was supported by the Research to Prevent Blindness Inc.

\section{References}

I Ramsay RC. Kinvoun JL. Hill CW. Aturaliva UP. Knobloch WH. Retinal astrocvtoma. Am J Ophthalmol 1979: 88: 32-6.

2 Jordano J. Galera H. Toro M. Carrieras B. Astrocvtoma of the retina. Br J Ophthalmol 1974: 58: 555-9.

3 Yanoff M. Zimmerman LE. Davis RL. Massive Gliosis of the retina. Int Ophthalmol Clin 1971: 11: 211-29.

+ Foos RY. Straatsma R. Allen RA. Astrocutoma of the optic nerve head. Arch Ophthalmol 1965: 74: 319-26.

5 Oehmichen M. Extracerebrale Gliome in Gesichtshereich. Acta Neuropathol (Berl) 1971: 17: 321-30. 


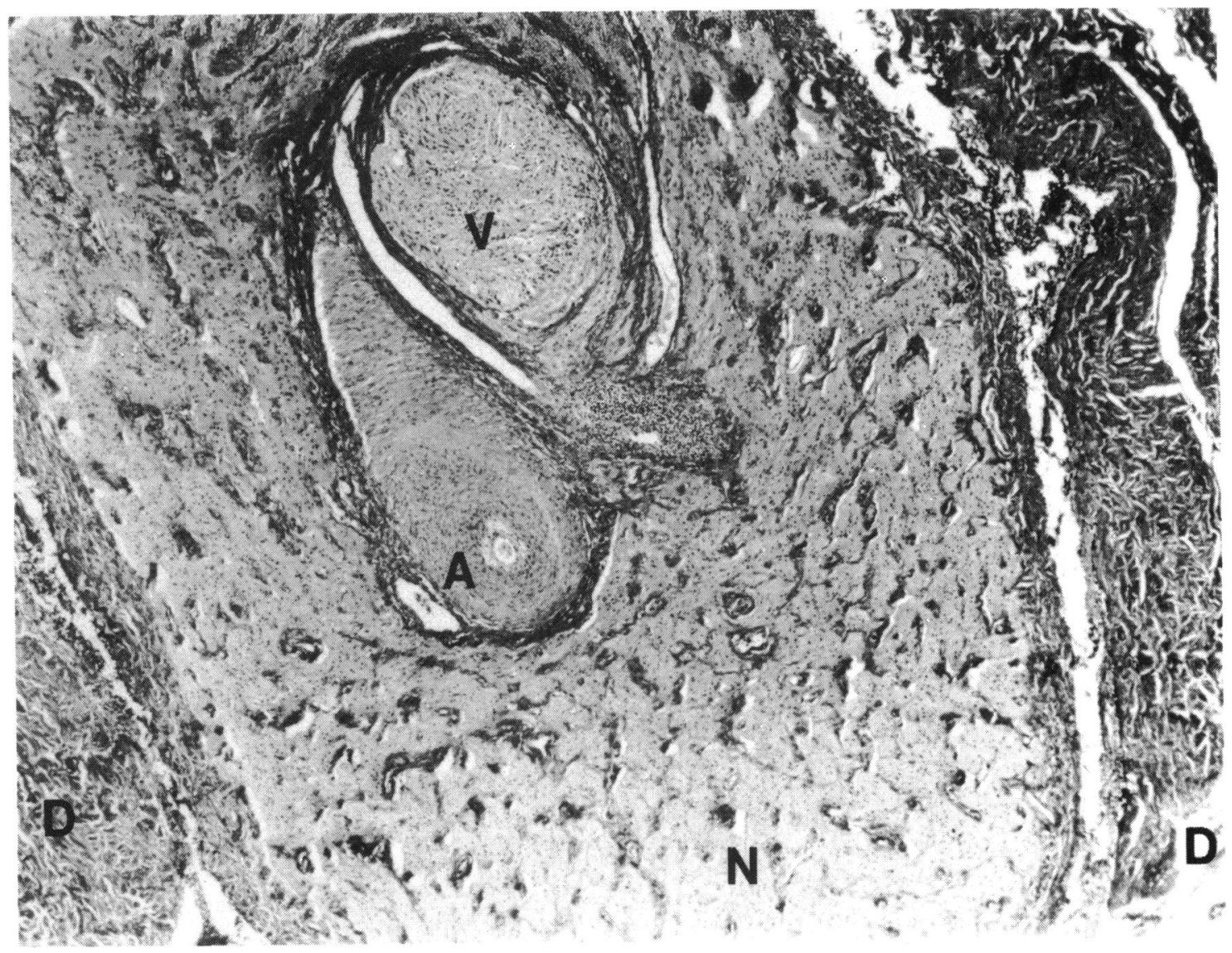

Fig. 6 Cross-section of the atrophic optic nerve stump $(\mathrm{N})$ with an extremely narrowed artery ( $\mathrm{A}$ ) and an organised thrombus in the central vein (V). No neoplasm is present at this level. The dura (D) is thickened. (Paraffin section, $H$ and $E$ stain. $\times 7.5)$.

6 Wolter JR. Better transillumination of enucleated eves with an egg candeler. Trans Am Ophthalmol Soc 1972: 70: 353-6.

7 Wolter JR. Brvson JM. Overabundance of uveal ganglion cells in eves with choroidal melanoma. Am J Ophthalmol 1966: 62: $1034-8$.

8 Maklev TA. King CM. Malignant melanoma of the choroid in melanosis oculi. Trans Am Acad Ophthalmol Otolarvngol 1967: 71: $6.38-41$. y Yanoff M. Zimmerman LE. Histogenesis of malignant melanomas of the uvea: III. The relationship of congenital ocular melanocytosis and neurofibromatosis to uveal melanoma. Arch Ophthalmol 1967: 77: 331-6.

10 Ogden TE. Nerve fiher laver astrocytes of the primate retina: morphologv. distribution and densitv. Invest Ophthalmol Visual Sci 1978: 17: 499-510. 